\title{
Analysis on a Common Fixed Point Theorem
}

\author{
V.Srinivas ${ }^{1}$, B.V.B.Reddy ${ }^{2} \&$ R.Umamaheshwar Rao ${ }^{3}$ \\ 1, 2.3 Department of Science and Humanities, Sreenidhi Institute of Science and Technology, Ghatkesar, \\ Hyderabad, India-501 301
}

\begin{abstract}
The aim of this paper is to prove a common fixed point theorem which generalizes the result of Brian Fisher [1] and etal. by weaker conditions. The conditions of continuity, compatibility and completeness of a metric space are replaced by weaker conditions such as reciprocally continuous and compatible, weakly compatible, and the associated sequence.
\end{abstract}

Keywords: Fixed point, self maps, reciprocally continuous, compatible maps, weakly compatible mappings.

\section{Introduction}

Two self maps $\mathrm{S}$ and $\mathrm{T}$ are said to be commutative if $\mathrm{ST}=\mathrm{TS}$. The concept of the commutativity has been generalized in several ways. For this Gerald Jungck [2] initiated the concept of compatibility.

\subsection{Compatible Mappings.}

Two self maps $\mathrm{S}$ and $\mathrm{T}$ of a metric space (X,d) are said to be compatible mappings if $\lim _{\mathbf{n} \rightarrow \infty}$ $d\left(\operatorname{STx}_{n}, T S x_{n}\right)=0$, whenever $\left\langle x_{n}>\right.$ is a sequence in $X$ such that $\lim _{n \rightarrow \infty} S_{x_{n}}=\operatorname{Tx}_{n}=t$ for some $t \in X$.

It can be easily verified that when the two mappings are commuting then they are compatible but not conversely.

In 1998, Jungck and Rhoades [4] introduced the notion of weakly compatible and showed that compatible maps are weakly compatible but not conversely.

\subsection{Weakly Compatible.}

A pair of maps $\mathrm{A}$ and $\mathrm{S}$ is called weakly compatible pair if they commute at coincidence points.

Brian Fisher and others [1] proved the following common Fixed Point theorem for four self maps of a complete metric space.

Theorem 1.3 Suppose A, B, S and T are four self maps of metric space(X,d)such that

1.3.1 (X,d) is a complete metric space

1.3.2 $\mathrm{A}(\mathrm{x}) \subseteq \mathrm{T}(\mathrm{x}), \mathrm{B}(\mathrm{x}) \subseteq \mathrm{S}(\mathrm{x})$

1.3.3 The pairs $(\mathrm{A}, \mathrm{S})$ and, $(\mathrm{B}, \mathrm{T})$ are compatible

1.3.4 $\mathrm{d}(\mathrm{Ax}, \mathrm{By})^{2}<\mathrm{c}_{1} \max \left\{\mathrm{d}(\mathrm{Sx}, \mathrm{Ax})^{2}, \mathrm{~d}(\mathrm{Ty}, \mathrm{By})^{2}, \mathrm{~d}(\mathrm{Sx}, \mathrm{Ty})^{2}\right\} \quad+\mathrm{c}_{2} \quad \max \{\mathrm{d}(\mathrm{Sx}, \mathrm{Ax}), \quad \mathrm{d}(\mathrm{Sx}, \mathrm{By})$, $\mathrm{d}(\mathrm{Ax}, \mathrm{Ty}), \mathrm{d}(\mathrm{By}, \mathrm{Ty})\}+\mathrm{c}_{3}\{\mathrm{~d}(\mathrm{Sx}, \mathrm{By}), \mathrm{d}(\mathrm{Ty}, \mathrm{Ax})\}$

Where $c_{1}, c_{2}, c_{3} \geq 0, c_{1}+2 c_{2}<1$ and $c_{1}+c_{3}>1$, then $A, B, S$ and $T$ have a unique common fixed point $z \in X$.

\subsection{Associated Sequence.}

Suppose A, B, S and T are self maps of a metric space (X, d) satisfying the condition (1.3.2), Then for any $\mathrm{x}_{0} \in \mathrm{X}, \mathrm{Ax} \mathrm{x}_{0} \in \mathrm{A}(\mathrm{X})$ and hence, $A \mathrm{x}_{0} \in \mathrm{T}(\mathrm{X})$ so that there is a $\mathrm{x}_{1} \in \mathrm{X}$ with $\mathrm{Ax}_{0}=\mathrm{Tx}_{1}$. Now $B \mathrm{x}_{1} \in \mathrm{B}(\mathrm{X})$ and hence there is $\mathrm{x}_{2} \in \mathrm{X}$ with $\mathrm{Bx}_{1}=\mathrm{Sx}_{2}$. Repeating this process to each $\mathrm{x}_{0} \in \mathrm{X}$, we get a sequence $\left\langle\mathrm{x}_{\mathrm{n}}>\right.$ in $\mathrm{X}$ such that $\mathrm{Ax}_{2 \mathrm{n}}=$ $\mathrm{Tx}_{2 \mathrm{n}+1}$ and $\mathrm{Bx}_{2 \mathrm{n}+1}=\mathrm{Sx}_{2 \mathrm{n}+2}$ for $\mathrm{n} \geq 0$. We shall call this sequence as an associated sequence of $\mathrm{x}_{0}$ relative to the Four self maps A, B,S and T.

Now we prove a lemma which plays an important role in proving our theorem.

1.5 Lemma. Suppose A, B, S and T are four self maps of a metric space (X, d) satisfying the conditions (1.3.2) and (1.3.4) of Theorem(1.3) and Further if (1.3.1) $\quad(\mathrm{X}, \mathrm{d})$ is a complete metric space then for any $\mathrm{x}_{0} \in \mathrm{X}$ and for any of its associated sequence $\left\langle\mathrm{x}_{\mathrm{n}}>\right.$ relative to Four self maps, the sequence $\mathrm{Ax}_{0}, \mathrm{Bx}_{1}, \mathrm{Ax}_{2}, \mathrm{Bx}_{3}$, ........, $\mathrm{Ax}_{2 \mathrm{n}}, \mathrm{Bx}_{2 \mathrm{n}+1}, \ldots \ldots \ldots$, converges to some point $\mathrm{z} \in \mathrm{X}$. 
Proof: For simplicity let us take $d_{n}=d\left(y_{n}, y_{n+1}\right)$ for $n=0,1,2, \ldots \ldots \ldots$

We have

$$
\begin{aligned}
& d^{2}{ }_{2 n+1}=\left[d\left(y_{2 n+1}, y_{2 n+2}\right)\right]^{2}=\left[d\left(A_{2 n}, B x_{2 n+1}\right)\right]^{2} \\
& \left.\leq \mathrm{c}_{1} \max \left\{\left[\mathrm{d}\left(\mathrm{Sx}_{2 \mathrm{n}}, \mathrm{Ax}_{2 \mathrm{n}}\right)\right]^{2}, \mathrm{~d}\left(\mathrm{Tx}_{2 \mathrm{n}+1}, \mathrm{Bx}_{2 \mathrm{n}+1}\right)\right]^{2},\left[\mathrm{~d}\left(\mathrm{Sx}_{2 \mathrm{n}}, \mathrm{Tx}_{2 \mathrm{n}+1}\right)\right]^{2}\right\} \\
& +\mathrm{c}_{2} \max \left\{\mathrm{d}\left(\mathrm{Sx}_{2 \mathrm{n}}, \mathrm{Ax}_{2 \mathrm{n}}\right), \mathrm{d}\left(\mathrm{Sx}_{2 \mathrm{n}}, \mathrm{Bx}_{2 \mathrm{n}+1}\right), \mathrm{d}\left(\mathrm{Ax}_{2 \mathrm{n}}, \mathrm{Tx}_{2 \mathrm{n}+1}\right) \mathrm{d}\left(\mathrm{Bx}_{2 \mathrm{n}+1}, \mathrm{Tx}_{2 \mathrm{n}+1}\right)\right\} \\
& +\mathrm{c}_{3} \max \left\{\mathrm{d}\left(\mathrm{Sx}_{2 \mathrm{n}}, \mathrm{Bx}_{2 \mathrm{n}+1}\right), \mathrm{d}\left(\mathrm{Tx}_{2 \mathrm{n}+1}, \mathrm{Ax}_{2 \mathrm{n}}\right)\right\} \\
& \leq \mathrm{c}_{1} \max \left\{\mathrm{d}_{2 \mathrm{n}}^{2}, \mathrm{~d}^{2}{ }_{2 \mathrm{n}+1}\right\}+\mathrm{c}_{2}\left\{\mathrm{~d}_{2 \mathrm{n}} \mathrm{d}\left(\mathrm{y}_{2 \mathrm{n}}, \mathrm{y}_{2 \mathrm{n}-2}\right)\right\} \\
& \leq \mathrm{c}_{1} \max \left\{\mathrm{d}^{2}{ }_{2 \mathrm{n}}, \mathrm{d}^{2}{ }_{2 \mathrm{n}+1}\right\}+\mathrm{c}_{2}\left[\mathrm{~d}_{2 \mathrm{n}}^{2}+\mathrm{d}_{2 \mathrm{n}} \mathrm{d}_{2 \mathrm{n}-1}\right] \\
& \leq \mathrm{c}_{1} \max \left\{\mathrm{d}^{2}{ }_{2 \mathrm{n}}, \mathrm{d}^{2}{ }_{2 \mathrm{n}+1}\right\}+\mathrm{c}_{2}\left[\frac{3}{2} \mathrm{~d}_{2 \mathrm{n}}^{2}+\frac{1}{2} \mathrm{~d}^{2}{ }_{2 \mathrm{n}-1}\right]
\end{aligned}
$$

If $\mathrm{d}_{2 \mathrm{n}+1}>\mathrm{d}_{2 \mathrm{n}}$, inequality (1.5.1) implies $\mathrm{d}^{2}{ }_{2 \mathrm{n}+1} \leq \frac{2 \mathrm{c} 2}{2-2 \mathrm{c} 1-\mathrm{c} 2} \mathrm{~d}^{2}{ }_{2 \mathrm{n}}$ a contradiction, since $\frac{3 \mathrm{c} 2}{2-2 \mathrm{c} 1-\mathrm{c} 2}<1$. Thus $\mathrm{d}_{2 \mathrm{n}+1} \leq \mathrm{d}_{2 \mathrm{n}}$ and inequality (1.5.1) implies that $\mathrm{d}_{2 \mathrm{n}+1}=\mathrm{d}\left(\mathrm{y}_{2 \mathrm{n}+1}, \mathrm{y}_{2 \mathrm{n}-2}\right) \leq \mathrm{hd}\left(\mathrm{y}_{2 \mathrm{n}}, \mathrm{y}_{2 \mathrm{n}+1}\right)=\mathrm{h}^{2} \mathrm{~d}_{2 \mathrm{n}}$ Where $\quad \mathrm{h}^{2}=\frac{2 \mathrm{c} 1+3 \mathrm{c} 2}{2-\mathrm{c} 2}<1$. Similarly, $\mathrm{d}_{2 \mathrm{n}}{ }^{2}=\left[\mathrm{d}\left(\mathrm{y}_{2 \mathrm{n}}, \mathrm{y}_{2 \mathrm{n}+1}\right]^{2}=\left[\mathrm{d}\left(\mathrm{Ax}_{2 \mathrm{n}}, \mathrm{Bx}_{2 \mathrm{n}-1}\right)\right]^{2} \leq \mathrm{c}_{1} \max \left\{\mathrm{d}_{2 \mathrm{n}-1}^{2}, \mathrm{~d}_{2 \mathrm{n}}^{2}\right\}+\mathrm{c}_{2}\left(\frac{3}{2} \mathrm{~d}_{2 \mathrm{n}-1}{ }^{2}+\frac{1}{2} \mathrm{~d}_{2 \mathrm{n}}^{2}\right)\right.$ and it follows above that $\mathrm{d}_{2 \mathrm{n}}=\mathrm{d}\left(\mathrm{y}_{2 \mathrm{n}}, \mathrm{y}_{2 \mathrm{n}+1}\right) \leq \mathrm{hd}\left(\mathrm{y}_{2 \mathrm{n}-1}, \mathrm{y}_{2 \mathrm{n}}\right)=\mathrm{d}_{2 \mathrm{n}-1}$

Consequently, $d\left(y_{n+1}, y_{n}\right) \leq h d\left(y_{n}, y_{n-1}\right)$, For $n=1,2,3 \ldots \ldots$ since $h<1$, this implies that $\left\{y_{n}\right\}$ is a cauchy sequence in $\mathrm{X}$.

Hence the Lemma.

The converse of the lemma is not true.

That is, suppose A, B, S and T are self maps of a metric space (X, d) satisfying the conditions (1.3.2) and (1.3.4), even for each associated sequence $\left\langle\mathrm{x}_{\mathrm{n}}\right\rangle$ of $\mathrm{x}_{0}$, the associated sequence converges, the metric space $(\mathrm{X}, \mathrm{d})$ need not be complete. For this we provide an example.

1.6 Example. Let $\mathrm{X}=(-1,1)$ with $\mathrm{d}(\mathrm{x}, \mathrm{y})=|x-y|$

$$
A x=B x=\left\{\begin{array} { l l } 
{ \frac { 1 } { 5 } } & { \text { if } - 1 < x < \frac { 1 } { 6 } } \\
{ \frac { 1 } { 6 } } & { \text { if } \frac { 1 } { 6 } \leq x < 1 }
\end{array} \text { if } \left\{x=\left\{\begin{array} { l l } 
{ \frac { 1 } { 5 } } & { \text { if } - 1 < x < \frac { 1 } { 6 } } \\
{ \frac { 6 x + 5 } { 3 6 } } & { \text { if } \frac { 1 } { 6 } \leq x < 1 }
\end{array} \text { Tx } \left\{\begin{array}{ll}
\frac{1}{5} & \text { if }-1<x<\frac{1}{6} \\
\frac{1}{3}-x & \text { if } \frac{1}{6} \leq x<1
\end{array}\right.\right.\right.\right.
$$

Then $\mathrm{A}(\mathrm{X})=\mathrm{B}(\mathrm{X})=\left\{\frac{1}{5}, \frac{1}{6}\right\}$ while $\mathrm{S}(\mathrm{X})==\left\{\frac{1}{5} \cup\left[\frac{1}{6}, \frac{11}{36}\right)\right\}, \mathrm{T}(\mathrm{X})=\left\{\frac{1}{5} \cup\left[\frac{1}{6}, \frac{-2}{3}\right)\right\}$ so that $\mathrm{A}(\mathrm{X}) \subset \mathrm{T}(\mathrm{X})$ and $\mathrm{B}(\mathrm{X}) \subset \mathrm{S}(\mathrm{X})$ proving the condition (1.3.2)of Theorem (1.3). Clearly $(X, d)$ is not a complete metric space. It is easy to prove that the associated sequence $\mathrm{Ax}_{0}, \mathrm{Bx}_{1}, \mathrm{Ax}_{2}, \mathrm{Bx}_{3}, . ., \mathrm{Ax}_{2 \mathrm{n}}, \mathrm{Bx}_{2 \mathrm{n}+1} \ldots$, converges to $\frac{1}{5}$ if $-1<x<\frac{1}{6}$; and converges to $\frac{1}{6}$, if $\frac{1}{6} \leq x<1$.

\section{Main Result}

Theorem 2.Suppose A, B, S and T are four self maps of metric space(X,d)such that $2.1 \quad \mathrm{~A}(\mathrm{x}) \subseteq \mathrm{T}(\mathrm{x}), \mathrm{B}(\mathrm{x}) \subseteq \mathrm{S}(\mathrm{x})$,

2.2 The pair $(\mathrm{A}, \mathrm{S})$ is reciprocally continuous and compatible, and the pair $(\mathrm{B}, \mathrm{T})$ is weakly compatible.

$2.3 \mathrm{~d}(\mathrm{Ax}, \mathrm{By})^{2}<\mathrm{c}_{1} \max \left\{\mathrm{d}(\mathrm{Sx}, \mathrm{Ax})^{2}, \mathrm{~d}(\mathrm{Ty}, \mathrm{By})^{2}, \mathrm{~d}(\mathrm{Sx}, \mathrm{Ty})^{2}\right\}+\mathrm{c}_{2} \max \{\mathrm{d}(\mathrm{Sx}, \mathrm{Ax}), \mathrm{d}(\mathrm{Sx}, \mathrm{By})$, $\mathrm{d}(\mathrm{Ax}, \mathrm{Ty}), \mathrm{d}(\mathrm{By}, \mathrm{Ty})\}+\mathrm{c}_{3}$

$\{\mathrm{d}(\mathrm{Sx}, \mathrm{By}), \mathrm{d}(\mathrm{Ty}, \mathrm{Ax})\} \quad$ where $\mathrm{c}_{1}, \mathrm{c}_{2}, \mathrm{c}_{3}, \geq 0, \mathrm{c}_{1}+2 \mathrm{c}_{2}<1$ and $\mathrm{c}_{1}+\mathrm{c}_{3}>1$ 
Further if

2.4 The sequence $\mathrm{Ax}_{0}, \mathrm{Bx}_{1}, \mathrm{Ax}_{2}, \mathrm{Bx}_{3} \ldots \ldots \mathrm{Ax}_{2} \mathrm{n}, \mathrm{Bx}_{2 \mathrm{n}+1} \ldots$ converges to $\mathrm{z} \in \mathrm{X}$ then $\mathrm{A}, \mathrm{B}, \mathrm{S}$ and $\mathrm{T}$ have a unique common fixed point $\mathrm{z} \in \mathrm{X}$.

Proof: From condition IV, $\mathrm{Ax}_{2 \mathrm{n}}, \mathrm{Bx}_{2 \mathrm{n}+1}$ converges to $\mathrm{z}$ as $\mathrm{n} \rightarrow \infty$.

Since the pair $(\mathrm{A}, \mathrm{S})$ is reciprocally continuous means $\mathrm{ASx}_{2 \mathrm{n}}$ converges to $\mathrm{Az}$ and $\mathrm{SAx}_{2 \mathrm{n}}$ converges to $\mathrm{Sz}$ as $\mathrm{n} \rightarrow$ $\infty$.

Also since the pair (A,S) is compatible, we get $\lim _{n \rightarrow \infty} d\left(A S x_{2 n}, S A x_{2 n}\right)=0$ or d(Az,Sz)=0 or $\mathrm{Az}=\mathrm{Sz}$.

Now d $(\mathrm{Az}, \mathrm{z})^{2}=\mathrm{d}\left(\mathrm{Az}, \mathrm{Bx}_{2 \mathrm{n}+1}\right)^{2} \leq \mathrm{c}_{1} \max \left\{\left[\mathrm{d}(\mathrm{Sz}, \mathrm{Az})^{2}, \mathrm{~d}\left(\mathrm{Tx}_{2 \mathrm{n}+1}, \mathrm{Bx}_{2 \mathrm{n}+1}\right)^{2}, \mathrm{~d}\left(\mathrm{Sz}_{,} \mathrm{Tx}_{2 \mathrm{n}+1}\right)^{2}\right]\right\}+\mathrm{c}_{2} \max \{\mathrm{d}(\mathrm{Sz}, \mathrm{Az})$

$\left\{\mathrm{d}\left(\mathrm{Sz}, \mathrm{Bx} \mathrm{x}_{2 \mathrm{n}+1}\right), \mathrm{d}\left(\mathrm{Tx}_{2 \mathrm{n}+1}, \mathrm{Az}\right)\right\}$ $\left.\mathrm{d}\left(\mathrm{Sz}, \mathrm{Bx}_{2 \mathrm{n}+1}\right), \mathrm{d}\left(\mathrm{Az}, \mathrm{Tx}_{2 \mathrm{n}+1}\right), \mathrm{d}\left(\mathrm{Bx}_{2 \mathrm{n}+1}, \mathrm{Tx}_{2 \mathrm{n}}\right)\right\}+\mathrm{c}_{3}$

Letting $n \rightarrow \infty$, we get $d(A z, z)^{2} \leq c_{1} d(A z, z)^{2}+c_{3} d(A z, z)^{2}=\left(c_{1}+c_{3}\right) d(A z, z)^{2}$

This gives $d(A z, z)^{2}\left[1-\left(c_{1}+c_{3}\right)\right] \leq 0$.

Since $c_{1}+c_{3}<1$, we get $d[(A z, z)]^{2}=0$ or $A z=z$. Therefore $z=A z=S z$.

Also Since $\mathrm{A}(\mathrm{x}) \subseteq \mathrm{T}(\mathrm{x}) \exists \mathrm{u} \in \mathrm{x}$ such that $\mathrm{z}=\mathrm{Az}=\mathrm{Tu}$.

We prove $\mathrm{Bu}=\mathrm{Tu}$.

Consider $\mathrm{d}[(\mathrm{z} . \mathrm{Bu})]^{2}=[\mathrm{d}(\mathrm{Az}, \mathrm{Bu})]^{2} \leq \mathrm{c}_{1} \max \left\{\left[\mathrm{d}(\mathrm{Sz}, \mathrm{Az})^{2}, \mathrm{~d}(\mathrm{Tu}, \mathrm{Bu})^{2}, \mathrm{~d}(\mathrm{Sz}, \mathrm{Tu})^{2}\right]\right\}$

$\mathrm{d}(\mathrm{z}, \mathrm{Bu})^{2} \leq \mathrm{c}_{1}+\mathrm{c}_{3} \mathrm{~d}(\mathrm{z}, \mathrm{Bu})^{2}$

$+\mathrm{c}_{2} \max \{\mathrm{d}(\mathrm{Sz}, \mathrm{Az}), \mathrm{d}(\mathrm{Sz}, \mathrm{Bu}), \mathrm{d}(\mathrm{Az}, \mathrm{Tu}), \mathrm{d}(\mathrm{Bu}, \mathrm{Tz})\}+\mathrm{c}_{3}\{\mathrm{~d}(\mathrm{Sz}, \mathrm{Bu}), \mathrm{d}(\mathrm{Tu}, \mathrm{Az})\}$

$=\mathrm{c}_{1} \mathrm{~d}(\mathrm{z}, \mathrm{Bu})^{2}+\mathrm{c}_{3} \mathrm{~d}(\mathrm{z}, \mathrm{Bu})^{2}$

$\mathrm{d}(\mathrm{z}, \mathrm{Bu})^{2}\left[1-\left(\mathrm{c}_{1}+\mathrm{c}_{3}\right)\right] \leq 0$ since $\mathrm{c}_{1}+\mathrm{c}_{3}<1$, we get $\mathrm{d}(\mathrm{z}, \mathrm{Bu})^{2}=0$ or $\mathrm{Bu}=\mathrm{z}$.

Therefore $\mathrm{z}=\mathrm{Bu}=\mathrm{Tu}$.

Since the pair $(B, T)$ is weakly compatible and $z=B u=T u$, we get $d(B B u, T T u)=0$ or $B z=T z$.

Now consider $\mathrm{d}(\mathrm{z}, \mathrm{Bz})^{2}=\mathrm{d}(\mathrm{Az}, \mathrm{Bz})^{2} \leq \mathrm{c}_{1} \max \left\{\left[\mathrm{d}(\mathrm{Sz}, \mathrm{Az})^{2}, \mathrm{~d}(\mathrm{Tz}, \mathrm{Bz})^{2}, \mathrm{~d}(\mathrm{Sz}, \mathrm{Tz})^{2}\right]\right\}+\mathrm{c}_{2} \max \{\mathrm{d}(\mathrm{Sz}, \mathrm{Az}), \mathrm{d}(\mathrm{Sz}, \mathrm{Bz})$, $\mathrm{d}(\mathrm{Az}, \mathrm{Tz}), \mathrm{d}(\mathrm{Bz}, \mathrm{Tz})\}+\mathrm{c}_{3}\{\mathrm{~d}(\mathrm{Sz}, \mathrm{Bz}), \mathrm{d}(\mathrm{Tz}, \mathrm{Az})\}=\mathrm{c}_{1} \mathrm{~d}(\mathrm{z}, \mathrm{Bz})^{2}+\mathrm{c}_{3} \mathrm{~d}(\mathrm{z}, \mathrm{Bz})^{2}$.

This gives

$\mathrm{d}(\mathrm{z}, \mathrm{Bz})^{2} \leq\left(\mathrm{c}_{1}+\mathrm{c}_{3}\right) \mathrm{d}(\mathrm{z}, \mathrm{Bz})^{2}$

$\mathrm{d}(\mathrm{z}, \mathrm{Bz})^{2}\left[1-\left(\mathrm{c}_{1}+\mathrm{c}_{3}\right)\right] \leq 0$, since $\mathrm{c}_{1}+\mathrm{c}_{3}<1$, we get $\mathrm{d}(\mathrm{z}, \mathrm{Bz})^{2}=0$ or $\mathrm{z}=\mathrm{Bz}$.

Therefore $\mathrm{z}=\mathrm{Bz}=\mathrm{Tz}$

Since $\mathrm{z}=\mathrm{Az}=\mathrm{Bz}=\mathrm{Sz}=\mathrm{T} \mathrm{z}, \mathrm{z}$ is a common fixed point of $\mathrm{A}, \mathrm{B}, \mathrm{S}$ and $\mathrm{T}$.

The uniqueness of common fixed point can be easily proved

Now, we discuss our earlier example in the following two remarks to justify our result.

Remark 2.5: From the example given earlier, clearly the pair $(A, S)$ is reciprocally continuous, since if $x_{n}=$ $\left(\frac{1}{6}+\frac{1}{6^{n}}\right)$ for $\mathrm{n} \geq 1$, then $\lim _{n \rightarrow \infty} A x_{n}=\lim _{n \rightarrow \infty} S x_{n}=\frac{1}{6}$ and $\lim _{n \rightarrow \infty} A S x_{n}=\frac{1}{6}=A(t)$ also $\lim _{n \rightarrow \infty} S A x_{n}=\frac{1}{6}=S(t)$.But none of $A$ and $S$ are continuous. Also, since $\lim _{n \rightarrow \infty} d\left(A S x_{n}, S A x_{n}\right)=0$, the pair $(A, S)$ is compatible. Moreover the pair $(\mathrm{B}, \mathrm{T})$ is weakly compatible as they commute at coincident points $\frac{1}{5}$ and $\frac{1}{6}$. The contractive condition holds for the values of $c_{1}, c_{2}, c_{3}, \geq 0, c_{1}+2 c_{2}<1$ and $c_{1}+c_{3}>1$. Further $\frac{1}{6}$ is the unique common fixed point of $A, B, S$ and T.

Remark 2.6: Finally we conclude that from the earlier example, the mappings $A, B, S$ and $T$ are not continuous, the pair $(\mathrm{A}, \mathrm{S})$ is reciprocally continuous and compatible and $(\mathrm{B}, \mathrm{T})$ is weakly compatible. Also the associated sequence relative to the self maps $\mathrm{A}, \mathrm{B}, \mathrm{S}$ and $\mathrm{T}$ such that the sequence $\mathrm{Ax}_{0}, \mathrm{Bx}_{1}, \mathrm{Ax}_{2}, \mathrm{Bx}_{3}, \ldots \ldots \ldots$, $\mathrm{Ax}_{2 \mathrm{n}}, \mathrm{Bx}_{2 \mathrm{n}+1}, \ldots \ldots . .$. , converges to the point $\frac{1}{6} \in \mathrm{X}$, but the metric space $\mathrm{X}$ is not complete. Moreover, $\frac{1}{6}$ is the unique common fixed point of A, B, S and T. Hence, Theorem (2) is a generalization of Theorem (1.3). 


\section{References}

[1] B.Fisher and etal, "Common Fixed Point Theorems for compatible mappings", Internat.J. Math. \& Math. Sci, 3(1996), 451-456.

[2] G .Jungck, “Compatible Mappings and Common Fixed Points”, Inst. J. Math. Math. Sci .9(1986), 771-779.

[3] G.Jungck, B.E. Rhoades, Fixed Point for set valued Functions without Continuity, Indian.J. Pure.Appl.Math,3(1998), 227-238.

[4] G. Jungck., B.E. Rhoades, Fixed point for set valued functions without continuity, Indian J. Pure.Appl.Math, 3 (1998), 227-238.

[5] V.Srinivas, R.Umamaheshwar Rao ,A Fixed point Theorem for Weekly Compatible Mappings, Journal of Mathematical Sciences \& Engineering Applications, 1(2007),41-48.

[6] V.Srinivas, R.Umamaheshwar Rao, Common Fixed Point Theorem for Four Self Maps, International Journal of Mathematics Research, 2(2011),113-118. 